\title{
ENTREVISTA CON ESTÍBALIZ PÉREZ PÉREZ TECNOLOGÍAS DIGITALES PARA EL MEJORAMIENTO DEL PROCESO DE ENSEÑNANZA Y APRENDIZAJE
}

\section{INTERVIEW WITH ESTÍBALIZ PÉREZ PÉREZ DIGITAL TECHNOLOGIES FOR THE IMPROVEMENT OF THE TEACHING AND LEARNING PROCESS}

Fecha de recepción: 01/09/2017

Fecha de aceptación: 27/11/2017

Realizada por: Consejo Editorial.

Resumen: La presente entrevista tiene como objetivo analizar la importancia de las tecnologías digitales como herramienta para el mejoramiento del proceso de enseñanza y aprendizaje. Para Estíbaliz Pérez las tecnologías digitales pueden ser elementos de motivación para el estudiantado, ya que su uso es parte de la vida cotidiana del estudiante. Además, afirma que las tecnologías por sí solas no tienen capacidad de mejorar los procesos de enseñanza y aprendizaje, pues se requiere de un proceso de mediación docente que atienda a los principios básicos de calidad educativa, porque su uso debe ser pertinente, relevante y equitativo.

Palabras clave: Tecnologías digitales; enseñanza; aprendizaje; entrevista.

Abstract: The objective of this interview is to analyze the importance of digital technologies as a tool for improving the teaching and learning process. For Estíbaliz Pérez, digital technologies can be motivating elements for students, since the use of technology is part of the student's daily life. It also states that technologies alone do not have the capacity to improve teaching and learning processes, since a process of teacher mediation that meets basic principles of educational quality is required, since its use must be relevant, relevant and equitable.

Keywords: Digital technologies; teaching; learning; interview.

\section{Presentación}

Estíbaliz Pérez Pérez, es máster en Tecnología Educativa, graduada de la Universidad Estatal a Distancia (UNED), Licenciada en Pedagogía y Didáctica con una formación basada en la Enseñanza de los Estudios Sociales y Cívica de Secundaria, ambos grados obtenidos en la Universidad Nacional de Costa Rica. En el área académica, cuenta con especializaciones en los siguientes temas: Aprendizaje en línea -eLearning-, Aprendizaje Móvil -Mobile Learning- y Gerencia de la Innovación.

Desde el año 2011, se ha especializado en la formación continua de docentes, específicamente en las áreas de Innovación Educativa apoyada en el uso de Tecnologías Digitales y metodologías innovadoras de aprendizaje. La docente ha colaborado directamente con instancias como el Ministerio de Educación Pública y la Fundación Omar Dengo, entre otros 
Entrevista con Estíbaliz Pérez Pérez. Tecnologías digitales para el mejoramiento del proceso de enseñanza y aprendizaje

organismos. Desde el año 2013 administra la comunidad de docentes de Facebook, "Profes de Est. Sociales y Cívica CR". ${ }^{1}$ Actualmente, se desempeña como Gestora de Innovación y Tecnología Educativa en el Colegio de Licenciados y Profesores en Letras, Filosofía, Ciencias y Artes de Costa Rica (Colypro).

\section{1. ¿Cuál es la importancia del uso de las TIC dentro del aula?}

También conocidas como tecnologías digitales (TD), prefiero llamarlas así pues es más amplio el término y resultan de mucho valor en los procesos de enseñanza y aprendizaje por varios motivos:

- Son elementos de motivación para el estudiantado, para las nuevas generaciones, el uso de la tecnología es parte de su vida cotidiana, de allí que muchos les llamen "Generación Net" o "Nativos Digitales".

- Son recursos, que integrados pedagógicamente en el aula, tienen el potencial de generar motivación, pero sobre todo el desarrollo de habilidades para la vida, pues facilitan la construcción de conocimiento desde una perspectiva más realista: por ejemplo, en el caso de la Enseñanza de la Geografía con la herramienta Google Maps, aquellos procesos de aprendizaje en los cuales se tenía que partir de una realidad abstracta para aprender, hoy pueden ocurrir en tiempo real y con acceso a información validada y la cual refleja contextos vivos.

- Desde el punto de vista de la Política Educativa, los procesos educativos apoyados con tecnologías, al generar motivación e interés en el estudiantado, colaboran a la retención escolar y disminución de la deserción educativa.

- A nivel de gestión y control de los procesos educativos pueden facilitar mucho el trabajo del profesorado y del centro educativo, pues permiten sistematizar procesos y datos relevantes para la mejora educativa; ejemplo de ello son los registros electrónicos, los repositorios de información, las aulas virtuales -como Moodle y Edmodo- y las aplicaciones de comunicación con el hogar como Remind y ClassDojo.

\footnotetext{
${ }^{1}$ Véase: https://www.facebook.com/groups/ProfesESyCivCR/.
} 


\section{2. ¿Garantiza el uso de la tecnología digital el mejoramiento del proceso de enseñanza y aprendizaje?}

Siendo concreta, no. Las tecnologías por sí solas no tienen capacidad de mejorar los procesos de enseñanza y aprendizaje; pero es allí donde se rompe el famoso mito que decía "que las tecnologías desplazarían al profesorado". Para que los procesos educativos apoyados con TIC atiendan los principios básicos de calidad educativa -sugeridos por UNESCO, 2016-, su uso debe ser pertinente -Responder al ¿Para qué? -, relevante -procesos significativos, interesantes y atractivos para el estudiantado- y equitativo -accesible para todos y todas-.

En ese sentido, quien tiene el potencial para diseñar procesos de enseñanza y aprendizaje mejorados con el uso de tecnologías digitales es el profesorado.

\section{3. ¿Cuál es el mayor reto que enfrentan los docentes de Estudios Sociales y Educación Cívica, respecto a los uso de las TIC?}

Los retos vinculados al uso de tecnologías son muchos, puesto que la tecnología avanza más rápido que los procesos educativos. Entre los principales retos podría destacar:

- La necesidad urgente de lograr que los procesos de enseñanza y aprendizaje trasciendan lo "memorístico" y que el conocimiento fortalecido en los estudiantes pueda ser aplicado en situaciones del mundo real, la tecnología es el aliado ideal para ello.

- Otro gran reto pendiente es estar al día sobre las nuevas herramientas tecnológicas que nos provee el entorno educativo, por ejemplo para muchos profesionales de la educación resulta de mucho interés conocer sobre nuevas aplicaciones móviles o programas en línea. Es un hecho que como docentes tenemos responsabilidades dentro y fuera del horario, lo cual limita muchas veces nuestro tiempo para investigar y probar esas nuevas herramientas. Sin embargo, la "magia de innovar con tecnología" no está en conocer muchas herramientas, sino en aplicar pocas, pero desde los principios de la relevancia y la pertinencia.

- Un reto general para docentes de Estudios Sociales y Cívica pero también de otras materias es el que continuamos muy aislados, nuestro nicho de trabajo sigue siendo el 
Entrevista con Estíbaliz Pérez Pérez. Tecnologías digitales para el mejoramiento del proceso de enseñanza y aprendizaje

aula, y las paredes nos limitan. Requerimos trascender ese espacio físico y conectarnos con otros docentes; ya que solo así podremos ser más innovadores, recomiendo al gremio docente a conectarse por medio de las redes sociales y aprender en conjunto, pues así podremos tener más ideas, acceso a recursos y tendremos más posibilidades de lograr innovación educativa.

- Un tema reciente y de mucho valor es el cómo podemos integrar el uso de dispositivos móviles, especialmente celulares propiedad de los estudiantes en nuestras clases. Se requiere tiempo para experimentar y sistematizar el Cómo lograr que estos dispositivos sirvan más que para socializar y entretenerse y para dar paso a un uso de estos recursos como herramienta para la construcción de conocimiento.

En esa línea, se requiere una coordinación conjunta de la comunidad educativa, directores, docentes, encargados de familia y estudiantes. Espero que los y las docentes de Estudios Sociales podamos ser pioneros en una estrategia que el MEP se encuentra promoviendo desde el 2016 y que en definitiva no se va a detener.

4. Fernando Avendaño (2005) asegura que es importante que los futuros profesores "se preparen para enseñar con tecnologías haciendo tecnología" y que, en su formación, "puedan proponer aplicaciones adecuadas que den respuesta a las necesidades de contextos específicos". ¿Qué opina usted respecto a esto? ¿El uso de las TIC en el aula pone en debate la formación docente?

La propuesta de Fernando Avendaño es sin duda una de mis favoritas, ya que vino a brindar en el 2007 mucha claridad sobre lo que implicaba la digitalización en los procesos educativos, pasar del uso de la hoja y el lápiz, a contar con procesadores y ahora dispositivos móviles como tabletas y celulares en nuestras aulas.

Enseñar con tecnología no me parece el fin último que debe guiarnos. Se ha visto por ejemplo, que instituciones y sistemas educativos invierten mucho dinero en la compra o dotación de equipo como computadoras, pizarras inteligentes y tabletas; sin embargo, cuando estas llegan al aula quien está a cargo del proceso de enseñanza y aprendizaje "no sabe qué hacer con ellas".

Considero que más que hacer la tecnología, lo que demuestra la experiencia internacional y desde una perspectiva muy personal, es que lo importante de nuestra parte no es hacer tecnología, lo más relevante es que podamos ser profesionales críticos de los recursos que 
tenemos a nuestro alcance y qué nos instruyamos por medio de cursos, conversaciones con colegas o recursos gratuitos en la web sobre cuáles son los estándares mínimos que nos deben aportar las herramientas tecnológicas para integrarlas en el aula; por ejemplo, al seleccionar un video producido en YouTube, definir si el video cuenta con audio e información correcta; si su extensión es pertinente para generar motivación y retención de lo aprendido; determinar la calidad de la luz y la validación de su contenido tanto didáctico como desde el aspecto disciplinar.

Adicionalmente, el video, no debe ser "solo para observarlo", es ideal que el/la docente, plantee una actividad didáctica en la cual el estudiantado aplique lo aprendido a través de ese video, por medio de una situación real.

En unos años, esperaría, además, que antes de optar por producciones externas, el gremio docente $\mathrm{y}$, en específico, los y las docentes de Estudios Sociales, generemos nuestros propios recursos educativos, por ejemplo videos con sello nacional, lo que implicará un gran cambio educativo en el uso y percepción de las tecnologías "evolucionar de un papel como consumidores de información para ser productores de información: al crearla y compartirla".

5. ¿Qué recomendación le daría a los docentes de Estudios Sociales y Educación Cívica para mejorar la enseñanza de la asignatura aprovechando las TIC?

Recomiendo, para mejorar la enseñanza de los Estudios Sociales y Cívica, pero también para mejorar en general la calidad de la educación:

a) Me parece fundamental que cuando realicemos actividades de clase, donde innovemos y cambiemos nuestras formas de enseñar y aprender, específicamente con tecnologías, sistematicemos -documentemos-, lo que sucede tanto lo bueno como lo malo, esto nos permitirá tomar mejores decisiones para futuras aplicaciones y sería ideal compartirlo con colegas del gremio para favorecer una cultura de Innovación Educativa.

b) Como educadores definitivamente hemos estado "expuestos" a procesos de formación y ejecución de los procesos de enseñanza y aprendizaje muy rigurosos, en los cuales no había derecho a equivocarnos, pues solíamos ser “los dueños de la clase pero también del conocimiento". En la Sociedad del Conocimiento equivocarnos es parte fundamental de nuestro quehacer y de la mejora educativa; eso sí, partiendo de un principio de 
experimentación, pues cualquier proceso educativo que integre el uso de tecnologías digitales debe ser planificado, sin embargo nada garantiza que sus resultados vayan a ser perfectos o positivos en su primera implementación. Desde esta línea sugiero que como docentes perdamos el miedo a equivocarnos y experimentemos más. Si en nuestros intentos por innovar con tecnología, algo sale mal es porque es parte del proceso, pero recuerde tener un plan alternativo -en caso de que esa primera opción no salga bien- esto me parece fundamental para evitar la frustración y motivarnos a seguirlo intentando.

c) Apoyemos nuestro diseño didáctico de la mano de las sugerencias e intereses del estudiantado. Estudiantes tienen a la mano aplicaciones, videos y software que nosotros podemos integrar en nuestras clases, prestemos atención a los recursos que usan. Este consejo sin embargo tiene una doble interpretación: que integremos en los procesos de enseñanza y aprendizaje aquellas herramientas que son de interés y motivación, lo cual no implica que nuestros estudiantes "sepan más que nosotros" por ser nativos digitales, me gusta mucho un término que utiliza frecuentemente Francesc Pedró, Director de Política Educativa de UNESCO París: muchos de nuestros estudiantes, más que nativos digitales son "Huérfanos Digitales" y desde esa perspectiva, podemos afirmar que nuestros estudiantes saben cómo entretenerse y socializar, pero como adultos y docentes somos clave para orientarles en un uso responsable, seguro y productivo de las tecnologías.

d) Generemos planteamientos educativos en los cuales demos prioridad al desarrollo de habilidades antes que el dominio de contenidos, esto responde al llamado "Enfoque de Aprendizaje por Competencias", pero más allá de ello, lo importante es generar experiencias educativas que colaboren a que estudiantes apliquen los conocimientos aprendidos en situaciones de la vida real, esto colaborará a que el conocimiento sea trascendental y no se olvide el fin de semana luego de hacer el examen.

e) No esperemos a tener la última tecnología para comenzar a innovar o enseñar de forma distinta. Utilicemos aquello "que tenemos a la mano", tratemos de utilizar recursos offline y aprovechemos los recursos del estudiantado: "los dispositivos móviles" como celulares y tabletas, los cuales pueden ayudarnos a diversificar nuestro quehacer. Existen 
razones importantes por las cuales es importante "no esperar a la compra del equipo más reciente": los sistemas educativos están optando por desestimar las políticas de compra y dotación de equipo, para propiciar un uso de lo que todos tienen a la mano -por lo menos un número importante de estudiantes-, el uso del celular y priorizar la conectividad como recurso. Hay estudios y desde mi perspectiva puedo validarlo con experiencias de formación docente que muestran que aquellas personas que no innovan con lo que tienen a la mano y tienen oportunidad de hacerlo con equipo nuevo, tampoco lo hacen, todo está en nuestra voluntad de cambiar las cosas, además de desarrollar nuestra creatividad.

\section{Referencias bibliográficas}

Avendaño, Fernando. (2005). La cultura escrita ya no es lo que era. Letras, escritura, tecnologías y escuela. Santa Fe, Argentina: Editorial Homo Sapiens.

Ministerio de Educación Pública de Costa Rica. (2017). Normativa para el uso de dispositivos móviles, propiedad de los estudiantes en el centro educativo. San José, Costa Rica: MEP. Recuperado de: http://www.mep.go.cr/sites/default/files/Normativa_uso_moviles.pdf.

OREALC/UNESCO Santiago. (2016). Tecnologías Digitales al servicio de la calidad educativa: Una propuesta de cambio centrada en el aprendizaje para todos. Santiago, Chile: UNESCO. Recuperado de: http://unesdoc.unesco.org/images/0024/002451/245115S.pdf. 